\title{
X-RAY STANDING WAVES: A POWERFUL TOOL FOR INTERFACE STUDIES
}

\author{
S. Lagomarsino, F. Scarinci \\ Istituto Elettronica Stato Solido, CNR, V. Cineto Romano, 4200156 Rome, Italy \\ P. Castrucci \\ Phys. Dep., Univ. Camcrino, 62032 Camerino, Italy \\ and C. Giannini \\ CNRSM, 72023 Mesagne, Italy
}

\begin{abstract}
The X-ray standing wave technique has demonstrated in these last years to be a powerful method in the study of interfaces. In this paper the fundamentals of the technique will be given, together with examples of applications in the field of metal-semiconductor, of buried semiconductor-semiconductor interfaces and in structural studies of Langmuir-Blodgett films.
\end{abstract}

PACS numbers: 07.85.+n, 61.10.Lx, 68.35.-p

\section{Introduction}

In the early sixties Batterman [1] in his development of dynamical theory of diffraction put the basis of a new method, the X-ray standing wave (XSW) technique, which revealed, years later, full of potentialities in the structural studies of surfaces, interfaces and thin films. To understand the principle of formation of the X-ray standing wave in a crystalline matrix and how they can be used for structural studies, let us start with an X-ray plane wave impinging on a perfect crystal at the proper angle in order to have Bragg diffraction for a given set of lattice plane $(h k l)$. In these conditions, due to the interference between the incident and diffracted wave fields, the resulting electromagnetic (e.m.) field inside the crystal is a standing wave having the equintensity planes parallel to the diffracting planes and with their periodicity $[1,2]$. It is well known from dynamical theory of diffraction that the phase difference between incident and diffracted wave fields changes by $\pi$ when the crystal passes from the low angle to the high angle side of the diffraction curve. The phase shift causes a displacement of the standing wave with respect to the diffracting planes as a function of the rocking angle, as shown in Fig. 1a and 1b. The e.m. field nodes coincide with the diffracting planes at the 
a)

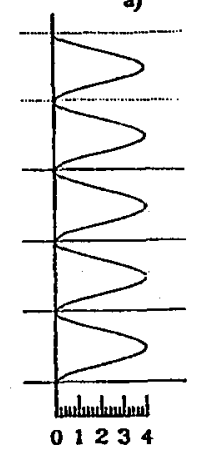

E.K. Field Intensity

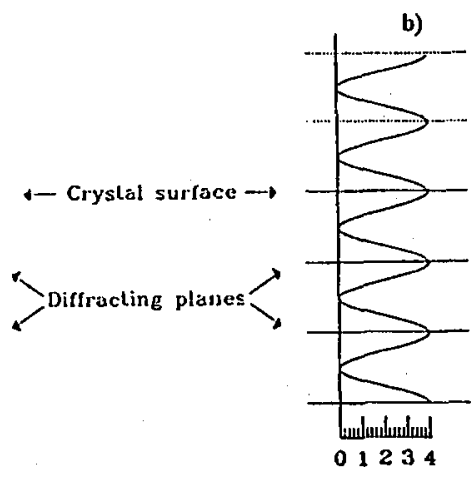

E.M. Field Intènsity

Fig. 1. Behaviour of the e.m. field intensity with respect to the diffracting planes in the low-angle (a) and high-angle (b) tails of the rocking curve.

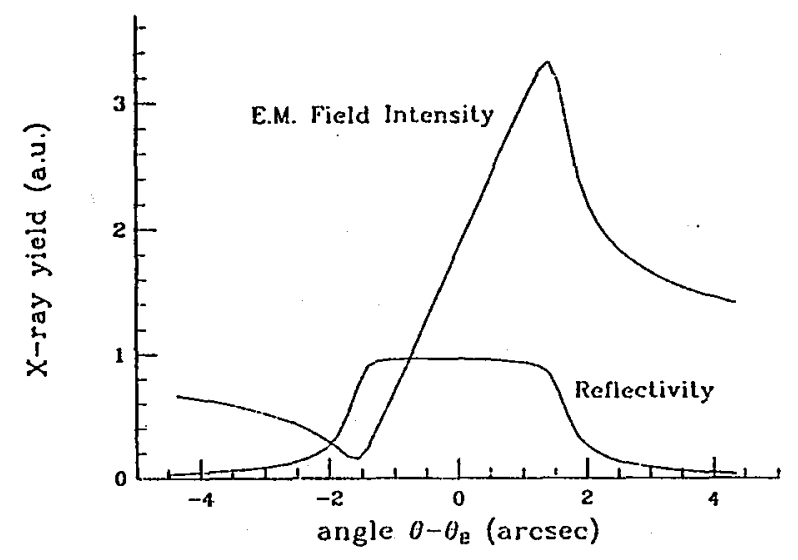

Fig. 2. Bragg reflectivity and e.m. field intensity as a function of the deviation from the Bragg angle.

low-angle side of the diffraction curve, while the antinodes coincide with them at the high-angle side. 'Therefore a modulation of the e.m. field intensity occurs when the crystal is rocked through its reflectivity curve, as illustrated in Fig. 2. This modulation can be revealed by measuring, as a function of the rocking angle, the yield of secondary events, e.g. fluorescence, plıotoemission, Auger emission, etc.

The key point to use the XSW ficld for structural studies is that the modulation is different for different points of the crystallographic unit cell. This is illustrated in Fig. 3a, b and $\mathrm{c}$ where the simulated fluorescence intensities resulting respectively from excited atoms at a distance $P$ from the difracting planes equal to the interplanar distance $d$, and from atoms at a distance $P=0.8 d$ and $P=0.6 d$ are shown. Dilferent and well distinguishable curves can be obtained for 


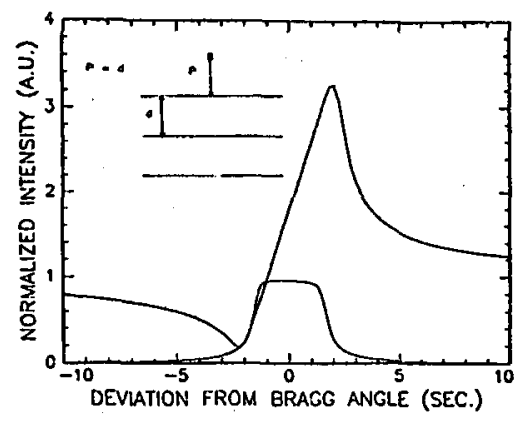

a)

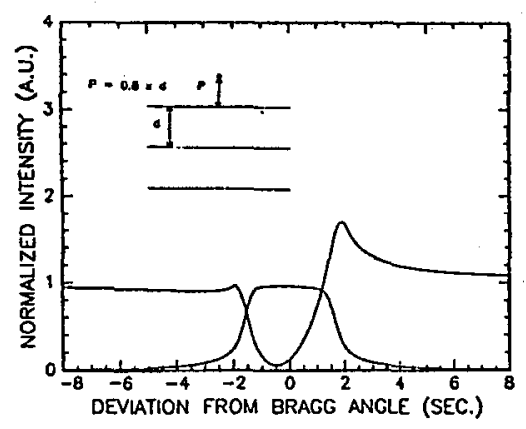

b)

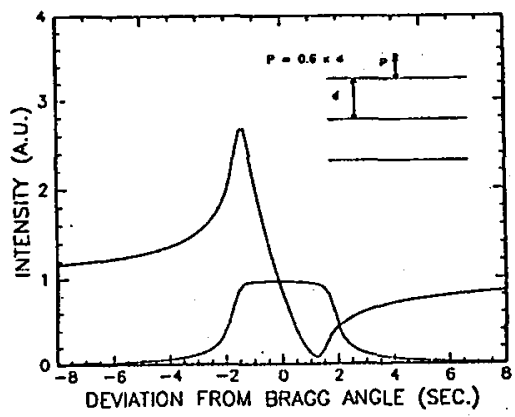

c)

Fig. 3. Calculated dependence of the fluorescence modulation as a function of the Bragg angle for three different positions of the excited atom with respect to the diffracting plane. $d$ is the XSW periodicily (equal to the interplanar spacing). (a) $P=d$; (b) $P=0.8 d$; (c) $P=0.6 d$.

intermediate values of $P$. It must be noted that in Fig. 3 the excited atoms are adsorbed on the substrate surface, and that the strong dependence of the modulation on the distance $P$ allows a very accurate determination of the adsorbate position with respect to the diffracting planes. The first evidence of the possibility to use the X-ray standing waves to obtain structural information on adsorbates was given by Cowan et al. in 1980 [3], opening a new and wide research field.

Equation (1) gives the intensity of the fluorescence (or of other decay signals) as a function of the rocking angle

$$
Y(0)=1+R(0)+2 F \sqrt{R(0)} \cos (\nu(0)-2 \pi P) .
$$


$R(\theta)$ is the reflectivity and $\nu(\theta)$ is the phase difference between the incident and the diffracted wave fields. These quantities are easily calculated from dynamical theory of diffraction.

$F$ and $P$ are, respectively, the amplitude and the phase of the charge density Fourier coefficient $F_{\mathrm{Xsw}}$ of the excited atoms whose decay is measured for the given reflection $(h k l)$ :

$$
F_{\mathrm{XsW}}=F_{h k l} \exp \left(2 \pi \mathrm{i} P_{h k l}\right) \text {. }
$$

If a percentage of the excited atoms occupies only one well defined position with respect to the diffracting planes $h k l$ and the rest of the atoms are randomly distributed, $P_{h k l}$ and $F_{h k l}$ represent respectively the actual position and the percentage of the total number of atoms in that position with respect to the $h k l$ diffracting planes. Ilowever, if a number of positions $p_{i}$ with relative occupation fractions $f_{i}$ are occupied, then the resulting $P$ and $F$ are related to the individual values $p_{i}$ and $f_{i}$ in the following way:

$$
\begin{aligned}
& \tan (2 \pi P)=\frac{\sum_{i} f_{i} \sin \left(2 \pi p_{i}\right)}{\sum_{i} f_{i} \cos \left(2 \pi p_{i}\right)}, \\
& F=f_{\text {com }} \exp (-M) \sqrt{\left(\sum_{i} f_{i} \sin \left(2 \pi p_{i}\right)\right)^{2}+\left(\sum_{i} f_{i} \cos \left(2 \pi p_{i}\right)\right)^{2}}
\end{aligned}
$$

The Debye-Waller factor $\exp (-M)$ and $f_{\text {com }}$ represent respectively the dynamic and static disorder. $f_{\text {com }}$ can liave values ranging between 0 and 1 , the smaller values indicating higher disorder.

The $P$ and $F$ values (named respectively "position" and "colherent fraction") are the free parameters in the fitting of the experimental curve (the decay signal intensity $Y(0)$ as a function of 0 ).

A complete structural determination requires in gencral the knowledge of the charge density Fourier component along different directions, therefore several non co-planar diffracting planes are needed, each set of planes giving the component along its perpendicular.

The accuracy that can be attained in the position determination is rather good: in favorable conditions an accuracy of the order of $0.01 \AA$ can be reached.

\section{Experimental requirements}

Previously, in the description of the formation of the XSW field in the crystal we made the assumption that a plane wave was incident on the crystal. In fact, a double-crystal diffractometer in parallel, i.e. non-dispersive, geometry is generally used. The experimental rocking curve is the result of the convolution between the intrinsic reflectivity curves of monochromators and sample. An asymmetrically cut monochromator is often used in order to reduce its intrinsic reflectivity curve width.

In XSW experiments the decay signal and the diffracted intensity must be simultaneously measured as a function of the Bragg angle. The rocking curve is in general very narrow (few seconds of arcs) and often the decay signal very weak, therefore long acquisition times (from few hours to some days) are required 
even with synchrotron racliation (at least with the presently a vailable sources). As a consequence, feedback stabilization systems are often used, based either on analog or digital teclnology $[4,5]$. When surface studies have to be carried out, both sample preparation and XSW measurements must be performed in ultrahigh vacuum. UIIV chambers for XSW experiments are installed in many synchrotron radiation facilities, with different layouts and different kind of detectors.

\section{Applications}

\subsection{Alkali metal/Si interface}

In this section we will give some results obtained in the study of alkali metal/Si interface. This kind of interfaces attracted recently great interest because of the special character of the interaction between the Si substrate atoms and the monovalent alkali metals. Interesting phenomena such as the large enhancement in the oxidation rate of the Si substrate when covered with alkali metals [6] and the ability of thin intralayers of alkali to control the band offsets in heterojunctions [7] have been observed. Despite this interest, the location of alkali atoms on the $\mathrm{Si}$ substrate is still an open question. We studied the $\mathrm{Rb} / \mathrm{Si}$ interface both on (111) and (100) orientations of the Si substrate in sub-monolayer coverage regime. In both cases we will show that in fact a complex situation with multisite occupancy takes place, and we will show how important in this case is to have information from several diffracting planes. The experiments were performed at the NSLS synchrotron facility in Brookhaven, where a UIIV beam line dedicated to XSW has been installed by $A T \& S T$. Rb was evaporated at room temperature on reconstructed $\mathrm{Si}$ surface: $7 \times 7$ for $\mathrm{Si}(111)$ and $2 \times 1$ for $\mathrm{Si}(100)$. Low energy electron diffraction (LEED) has been used to check the Si surface after reconstruction and after Rb deposition. In both the orientations sharp LEED patterns have been obtained after a chemical treatment (Shiraki etch) of the samples outside the vacuum chamber and a thermal treatment at about $800^{\circ} \mathrm{C}$ in vacuum. No new reconstruction structures have been detected by LEED after alkali metal deposition [8].

Coverage was measured by means of the Auger spectroscopy and X-ray fluorescence. Absolute coverage measurements were obtained with samples capped with $\mathrm{Si}$ and previously calibrated with Rutherford backscattering. Two coverages were studied in $\mathrm{Si}(111): 0.05 \mathrm{ML}$ (monolayer) and $0.16 \mathrm{ML}\left(1 \mathrm{ML}=7.83 \times 10^{14}\right.$ at./ $\mathrm{cm}^{2}$ ) while in Si(100) only one coverage was analyzed: $0.19 \mathrm{ML}$ (in this case 1 $\left.\mathrm{ML}=6.78 \times 10^{14} \mathrm{at} . / \mathrm{cm}^{2}\right)$. The $\mathrm{Rb} K_{\alpha}$ fluorescence line was measured, together with the difracted intensity. For clarity purposes discussion of the results will be given separately for the two orientations.

\subsection{1. $\operatorname{Si}(111)$}

In this case two reflections from different diffracting planes were used, i.e. (111) and (-111). The former set of planes is parallel to the surface, while the second one forms an angle of $70.54^{\circ}$ with the (111) surface, and gives therefore information about a different component. The alkali metals were evaporated on the 


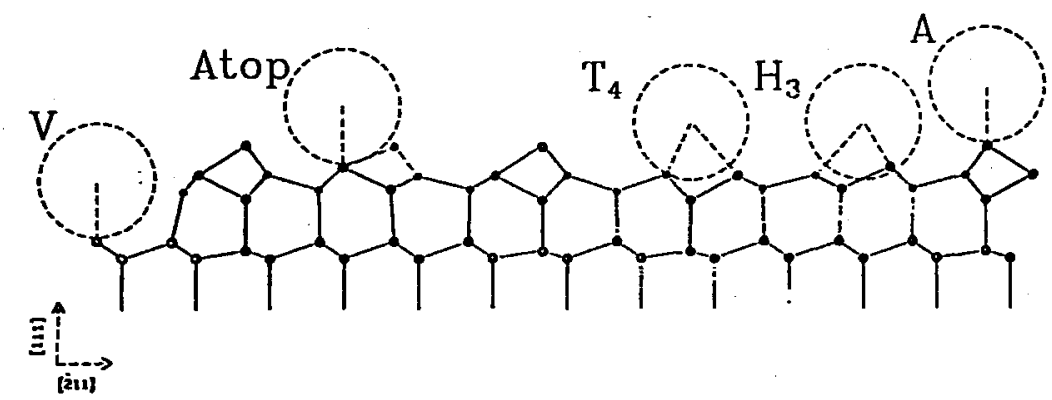

Fig. 4. (-111) side view of the $7 \times 7$ cell reconstructed according to the DAS model. The $H 3, T 4$, atop, adatoms $(A)$ and vacaucy $(V)$ sites are iudicated.

$7 \times 7$ reconstruction described by the D $\Lambda$ S (dimer-adatom stacking fault) model of Takayanagi [9], schematically shown in Fig. 4. The thrcefold hollow site' (H3), the threefold filled site (T4), the atop site, the adatom $(A)$ site and the vacancy site $(V)$ were considered as possible adsorption sites. Morcover, we distinguished among the faulted and the unfaulted halves of the reconstructed cell. The XSW experimental results are shown in Fig. 5a and 5b for the two diffracting planes. From these measurements four parameters are determined, two for each reflection: the positions $P_{111}$ and $P_{-111}$ and the coherent fractions $F_{111}$ and $F_{-111}$. If many sites are involved, as is the present case, two reflections are not enough to obtain directly, without any other hypothesis, the exact location of the adsorbed atoms. Therefore we calculated the position of the sites under consideration from the coordinates calculated for the $7 \times 7$ reconstruction by Qian and Chadi [10] and assuming that the bond length between Si and alkali atoms is the sum of the covalent radii. This assumption is based on both theoretical and experimental indications [11]. In this way it was possible to calculate the position $p_{i}$ for all the sites under consideration and for each set of diffracting planes. Allowing the occupation probability $f_{i}$ of each location $p_{i}$ to vary between 0 and 1 , all the possible site combinations were obtained. For each combination the $P$ and $F$ values are calculated from Eqs. (2) and (3) and then compared with the corresponding experimental quantities, taking into accomnt also the possible occurrence of static and dynamic disorder. Only the distributions giving a good agreement with the experiment are therefore taken into consideration. Table I gives the numerical results, while Fig. 6 shows the site distribution that we found. The results can be summarized as follows:

i) More than one site is simultaneously occupied by alkali atoms on $\mathrm{Si}(111)$ $7 \times 7$ surface.

ii) The alkali atoms for both coverages are mainly distributed among the H3 unfault, the T4 (it is not possible with XSIV to distinguish between T4 on the faulted and T4 on the unfaulted part of the cell) and the atop fault sites. Our measurement therefore clearly indicates a clear distinction in adsorption properties between the faulted and the unfaulted halves of the $7 \times 7$ reconstruction, as already reported by some authors [12]. 

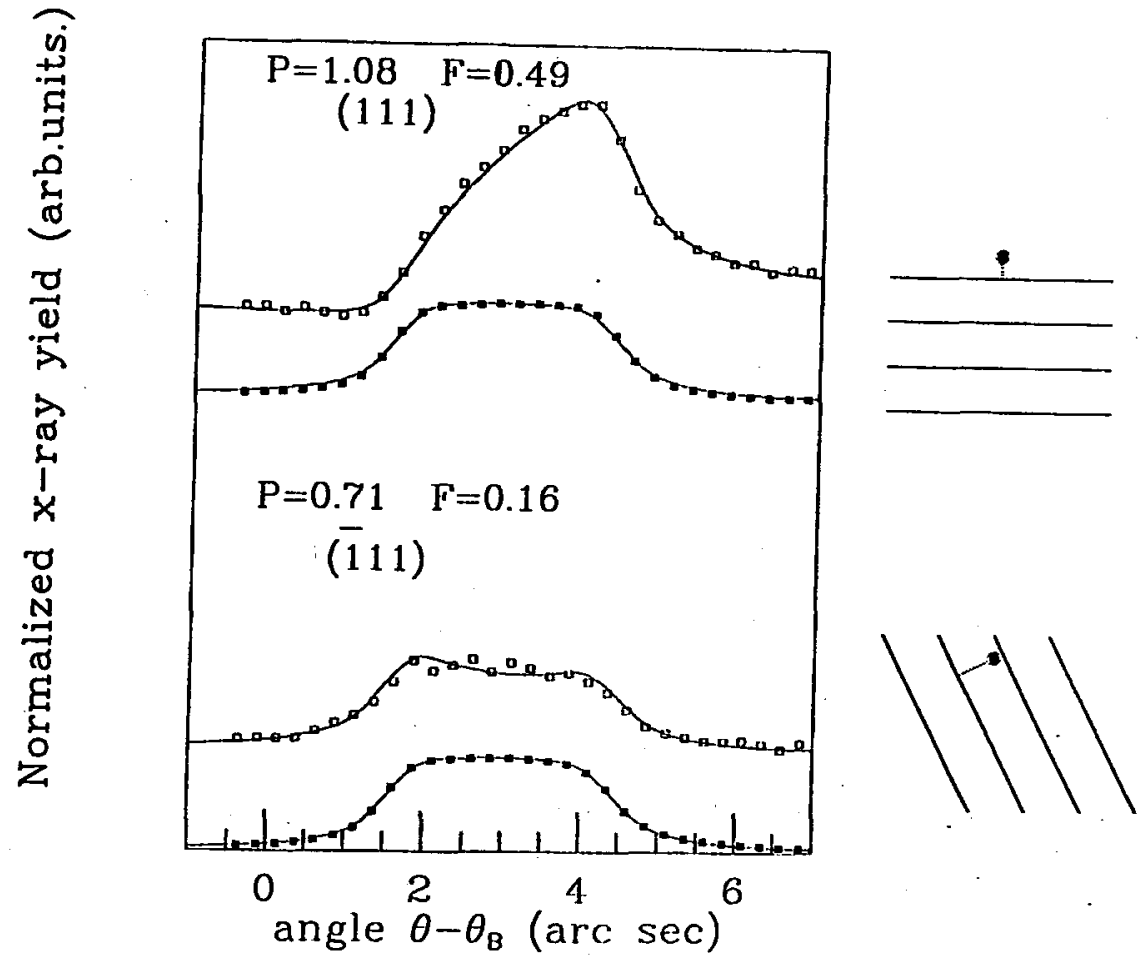

Fig. 5. XSW Rb results for (111) and (-111) diffracting planes. Open square: Auorescence yield. Filled square: diffracted intensity. Solid lines are theoretical fits to the experimental data.

TABLE I

Calculated and experimental results for (111) and (-111) diffracting planes.

\begin{tabular}{c|c|c|c|c|c|c}
\hline \hline & & $(111)$ & & & $(-111)$ & \\
\hline & Exp. & Calc. & $f_{\text {com }}$ & Exp. & Calc. & $f_{\text {com }}$ \\
\hline $0.05 \mathrm{ML}$ & $P=1.03$ & $P=1.02$ & & $P=0.74$ & $P=0.77$ & \\
& \pm 0.02 & \pm 0.02 & & \pm 0.04 & \pm 0.04 & \\
\hline & $F=0.33$ & $F=0.44$ & 0.75 & $F=0.22$ & $F=0.33$ & 0.69 \\
& \pm 0.05 & \pm 0.05 & \pm 0.08 & \pm 0.04 & \pm 0.05 & \pm 0.07 \\
\hline $0.16 \mathrm{ML}$ & $P=1.08$ & $P=1.07$ & & $P=0.71$ & $P=0.72$ & \\
& \pm 0.02 & \pm 0.02 & & \pm 0.04 & \pm 0.04 & \\
\hline & $F=0.49$ & $F=0.53$ & 0.93 & $F=0.16$ & $F=0.21$ & 0.76 \\
& \pm 0.05 & \pm 0.05 & \pm 0.09 & \pm 0.05 & \pm 0.05 & \pm 0.08
\end{tabular}




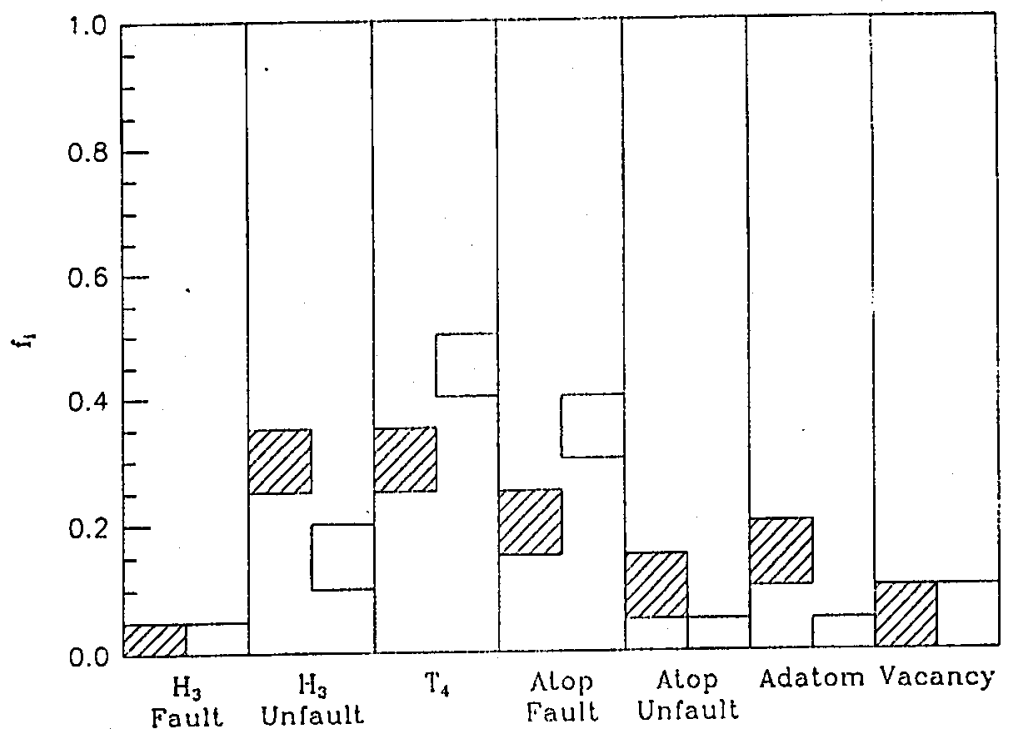

Fig. 6. Bar plot of the Rb site distributions giving a good agreement between the calculated and the experimental values of posilion and coliercut fraction. The hatched boxes refer to $0.05 \mathrm{ML}$ coverage, the white ones to $0.16 \mathrm{ML}$.

\subsection{2. $\mathrm{Si}(100)$}

As stated before, the Si(100) surface displayed the typical $(2 \times 1)$ reconstruction. It is known that the $2 \times 1$ reconstruction contains both asymmetric dimers and oscillating dimers whose lime-a veraged position is the symmetric one [13]. The orientation of dimers with respect to the crystallographic axes identifies two domains rotated of $90^{\circ}$ ones with respect to the others. From the LEED pattern we evaluated an equiprobable presence of the two domains. In order to have more complete information about the interface structure we used in this case three diffracting planes: (400), (311) and (220). The first plane is parallel to the surface, while the (311) and the (220) planes form respectively an angle of $25.14^{\circ}$ and $45^{\circ}$ with the surface. Figure $7 \mathrm{a}$ and $7 \mathrm{~b}$ shows the measured Rb fluorescence and diffracted intensity for the three reflections, and Table II summarizes the $P$. and $F$ values found. As in the case of (11.1) orientation the low value of the coherent fraction gives indication of a multisitc occupancy. Figure 8 shows the possible occupancy sites in the $(2 \times 1)$ surface cell. $A l l$ these sites, i.e. the bridge $(B)$, the pedestal $(P)$, the cave $(C)$, the valley $(V)$ and the site on top of the higher between the buckled dimers atoms were considered as possible adsorption sites. Moreover, we distinguished, by means of the (311) reflection, between the two possible arrangements of the buckled dimer, i.e. $T_{1}$ and $T_{2}$ (see Fig. 9). We assumed for symmetry reasons that adsorption at $B, P, C$ and $V$ sites symmetrizes the dimers. The procedure is now similar to the one adopted in the case of (111) orientation, but we have in this case three reflections instead of two, and therefore 
TABLE II

Experimental results for the colierent position $P$ and coherent fraction $F$ for the (004), (113) and (022) rellections for $0.19 \mathrm{ML} \mathrm{Rb}$ on $\mathrm{Si}(001)$.

\begin{tabular}{c|c|c}
\hline \hline$(h k l)$ & $P$ & $F$ \\
\hline$(004)$ & $0.23 \pm 0.03$ & $0.36 \pm 0.05$ \\
$(113)$ & $0.57 \pm 0.05$ & $0.09 \pm 0.05$ \\
$(022)$ & $0.50 \pm 0.05$ & $0.38 \pm 0.05$
\end{tabular}
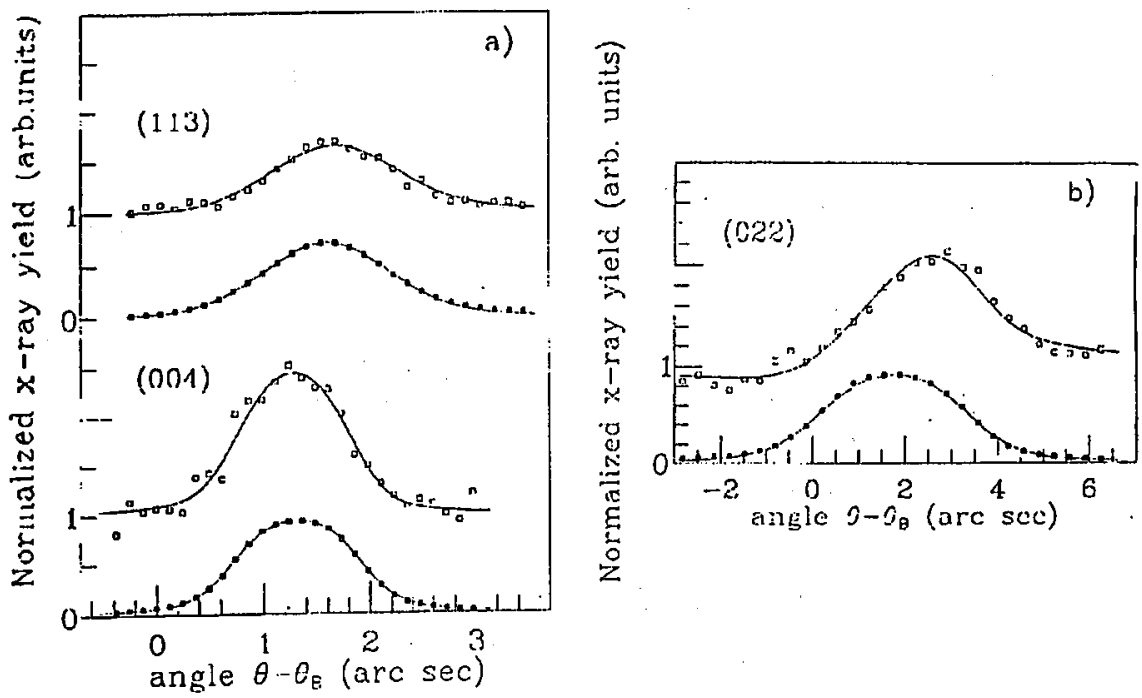

Fig. 7. XSW experimental results of $\mathrm{Rb} / \mathrm{Si}(001)$ interface. Coverage: 0.19 ML. (a) (113) and (004) reflections; (b) (022) reflection. Filled squares represent difracted intensity, open squares fluorescence intensily as a function of deviation from the Bragg angles. Solid lines are theoretical fits to the experimental data.

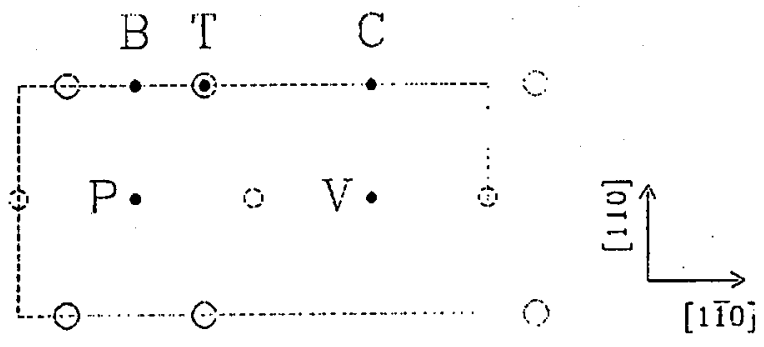

Fig. 8. Top view of the $\mathrm{Si}(001) 2 \times 1$ reconstructed surface. The pedestal $(P)$, bridge $(B)$, valley $(V)$, cave $(C)$ and top $(T)$ (sce text) are shown. 

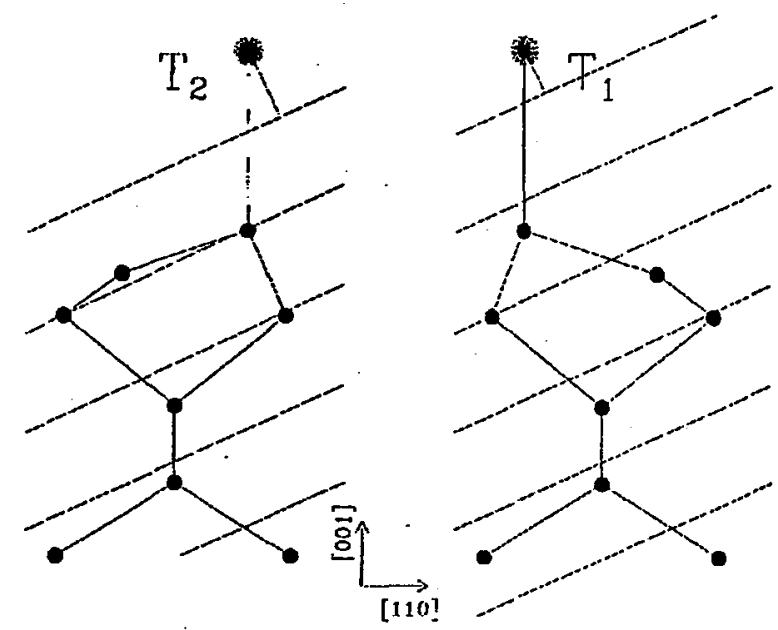

Fig. 9. $T_{1}$ and $T_{2}$ adsorption sites for a $\operatorname{Si}(0001)(1 \times 2)$ domain. The $(113)$ planes are indicated.

TABLE III

Adsorption site distribution obtained by analysis of the experimental data for $0.19 \mathrm{ML} \mathrm{Rb}$ adsorbed on $\mathrm{Si}(001) 2 \times 1$.

\begin{tabular}{l|r}
\hline \multicolumn{2}{c}{ Site distribution } \\
\hline Pedestal $(P)$ & $0.10 \pm 0.05$ \\
Bridge $(B)$ & $0.10 \pm 0.05$ \\
Cave $(C)$ & $0.00 \pm 0.05$ \\
Valley $(V)$ & $0.60 \pm 0.05$ \\
Top $T_{1}$ & $0.20 \pm 0.05$ \\
Top $T_{2}$ & $0.00 \pm 0.05$
\end{tabular}

we can determine a larger number of unknowns. In particular we did not fix the bond length between Si and alkali metal to a determined value, but we allowed it to vary between two limiting values, i.e. from the sum of the ionic radii to the sum of the covalent radii of $\mathrm{Si}$ and $\mathrm{Rb}$. In addition, we considered the possibility of a rearrangement of the $\mathrm{Si}$ outmost layer due to the $\mathrm{Rb}$ adsorption. Table III summarizes the site distribution we obtained. The large majority (60\%) of the $\mathrm{Rb}$ atoms adsorb at the valley site, $20 \%$ at top $T_{1}$ site and $10 \%$ at pedestal and bridge sites. The bond length turned out to be $3.06 \pm 0.03 \AA$, few tenths of an angstrom shorter than the sum of the covalent radii. $\Lambda$ significant rearrangement of the Si outmost layer due to Rb adsorption takes place. In fact, the Si atoms in the symmetric dimers move in both the perpendicular and parallel directions to a position which is close to the $1 \times 1$ structure. Adsorption on $T_{1}$ induces also a displacement of the higher Si atom of the dimer pair. 
In conclusion, the example of the $\mathrm{Si} /$ alkali metal interface shows that also in complicated surface structure where multisite occupancy takes place, the XSW technique can give quantitative information about atomic location, bond length and surface rearrangement. From the discussion it results how important is to use the largest possible number of reflections in order to obtain reliable and self-consistent results.

\subsection{GaAs buried inlerfaces}

A recent application of the XSW technique deals with the GaAs/InAs/GaAs interface where the problem of In segregalion is of primary importance. We studied [14] an InAs monolayer encapsulated between the GaAs substrate and a GaAs cap layer $300 \AA$ thick. The sanple was preparcd at the Max-Planck Institute in Stuttgart following a special procedure to limit In segregation. Iligh resolution $\mathrm{X}$-ray diffraction (IIRXRD) measurements yielded the total quantity of In atoms which turned out to be 1.1 ML, and confirmed us that the epitaxial In layer was coherent with the substrate. The XSIV measurements were performed on the beam line D15B of the DCI storage ring of the synchrotron source LURE in Orsay. The (400) reflection was recorded and the $I_{11} L_{\alpha}$ fluorescence intensity was measured as a function of the Bragg angle. 'The results are shown in Fig. 10. From the fitting of the experimental curve we obtained $P=1.17 \pm 0.02$ and $F=0.58 \pm 0.07$, where $P$ is the resulting position of In with respect to the GaAs diffracting planes and $F$ is the resulting coherent fraction. 'Taking into account the results of the IIRXRD we found a distribution of In atoms in three monolayers: $75 \%$ of the total In atoms are incorporated in the first monolayer, $20 \%$ in the second and $5 \%$ in the third one, as shown in Fig. 11. Morcover, we found that the In atoms are not randomly distributed as they would be in a $\mathrm{Ga}_{x} \mathrm{In}_{1-x}$ As alloy, but form InAs terraces. Each

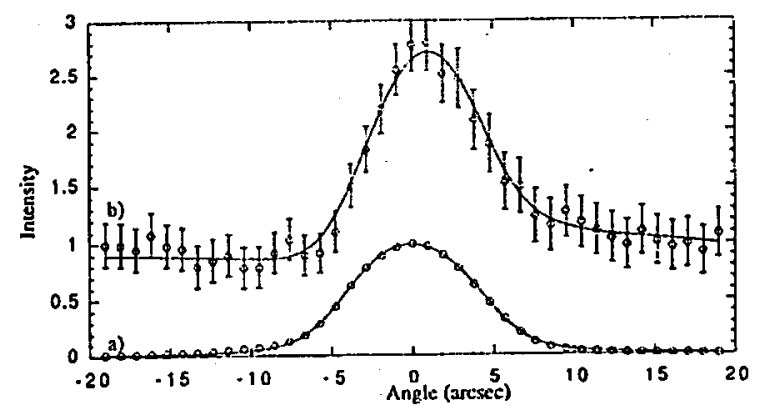

Fig. 10. Bragg reflectivity and fluorescence In $L_{\alpha}$ intensity (with error bars) as a function of deviation from the $B r a g g$ angle for a $\mathrm{Ga} \Lambda \mathrm{s} / \mathrm{hu} \Lambda \mathrm{s} / \mathrm{Ga} \Lambda$ s interface. Solid line is the theoretical fit. 


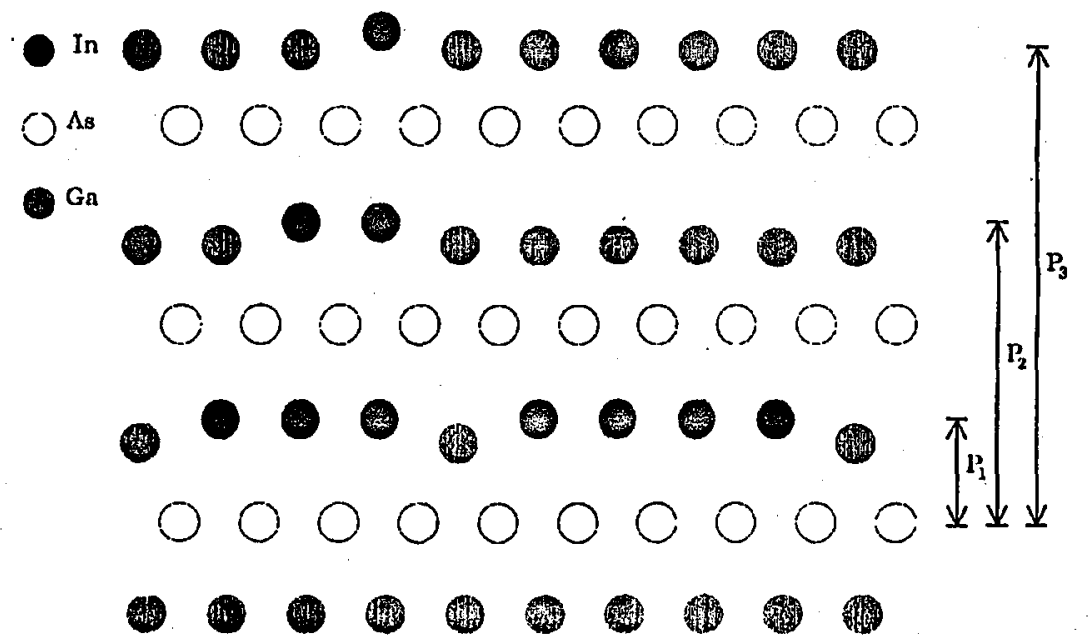

Fig. 11. Schematic arrangement of the In atoms with respect to $\mathrm{Ga}$ and As atoms at the interface. $P_{1}, P_{2}$ and $P_{3}$ are the distances of the In atoms in the first, second and third layers respectively.

position is displaced with respect to the GaAs planes by $15 \%$, as we would expect in the case of terraces colherent with the substrate and tetragonally deformed.

\section{Long period standing waves}

As mentioned above, the period of the standing waves is equal to the interplanar spacing $d$ of the diffracting planes. As a consequence, the formation of long-period standing waves liave been demonstrated in artificial superstructures [15] and in Langmuir-Blodgett films, these last consisting of organic molecules self-organised in woll defincd molecular laycrs $[16,17]$. The lattice spacing is in these cases of the order of few tchs of angstroms.

In Ref. [17] a Langmuir-Blodgett film consisting of about 100 monolayers of lead stearate deposited on a Si substrate and overcoated with one monolayer of barium behenate has been studied, recording the $\mathrm{Pb} M_{\alpha}$ and the Ba $L_{\alpha}$ fluorescence both in the angular region of the first order diffracted intensity and in the total reflection region. Figure 12 show's, respectively, the $\mathrm{Pb}$ and $\mathrm{Ba}$ fluorescence intensity as a function of incidence angle in the total reflection region, recorded with standard generator at the Institule.

The analysis of fluorescence modulation in the diffracted region is not dissimilar from what presented before for nalural crystals, in the sense that the standing waves are formed by the interference betwecn incident and diffracted waves and have the same period of the diffracting planes. The situation is different in the total reflection region. In this case, duc to the fact that the index of refraction in matter for X-rays is less than 1, under a critical angle the X-rays are totally reflected from a solid surface and standing waves whose period $d$ is a function of the incidence angle: $d=\lambda / 2 \sin 0$ ( $\lambda$ is the incident radiation wavelength) are generated above the reflecting surface [18]. 

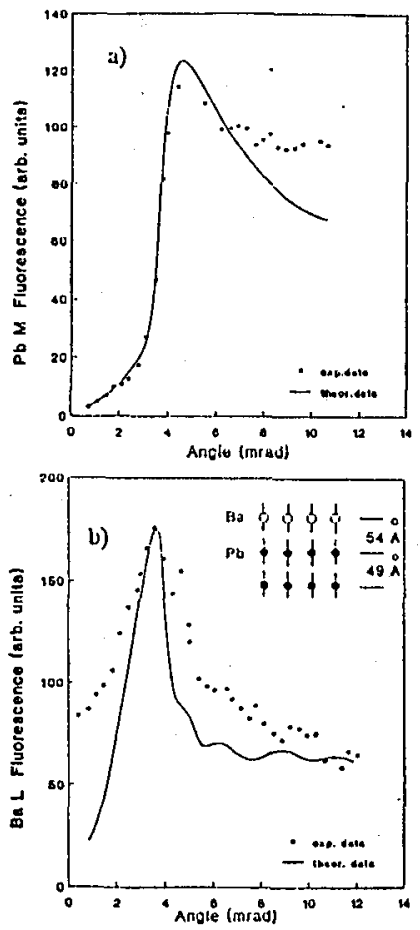

Fig. 12. Experimental and calculated fluorescence intensity in the angular region of total reflection for a sample composed of $100 \mathrm{ML}$ of Pb stearate on Si substrate overcoated with $1 \mathrm{ML}$ of $\mathrm{Ba}$ behenate. (a) Pb $M_{\mathrm{a}}$ flnorescence intensity. (b) Ba $L_{\alpha}$ fuorescence intensity.

Scanning the angle from zero to the critical angle makes the first antinodes to move from infinity to the mirror surface, with the period $d$ changing as a function of the angle. A probe placed at a certain distance $D$ from the surface will sense the movement of the antinodes and therefore will show modulation in the fluorescence (or other decay signals) intcnsity. The angular position of the maximum in fluorescence intensity is therclore related to the distance $D$ of the probe from the reflecting surface. $\mathrm{It}$ is worth to note that in Fig. 12 the maximum in $\mathrm{Ba}$ fluorescence is at a lower angle with respect to the maximum in $\mathrm{Pb}$ fluorescence, as expected from calculations [1T]. 'This method can give accurate atomic localization in the few tens to hundred angstrons range, opening very interesting perspectives in biomembrane studies and in biosensors.

In conclusion, the great potentialitics of the XSW technique have been put in evidence. These potentialities have not yct becn completely exploited, due to the complexity of the technicue and to the long acquisition time still needed in many cases. The new generation of synchrotron radiation sources will certainly constitute a breakthrough and a great opportunity for this fascinating tool for matter investigation. 


\section{References}

[1] B.W. Batterman, Pliys. Rev. A 133, 759 (1964).

[2] J.A. Golovchenko, B.W. Batterman, W.L. Brown, Phys. Rev. B 10, 4239 (1974).

[3] P.L. Cowan, J.A. Golovchenko, M.F. Robbins, Phys. Rev. Lett. 44, 1680 (1980).

[4] A. Krolzig, G. Materlik, J. Zegenhagen, Nucl. Instrum. Methods 208, 613 (1983).

[5] S. Lagomarsino, A. Nikolaenko, F. Scarinci, S. D'angelo, J. Derrien, J.Y. Veuillen, Nuovo Cimento D 10, 709 (1988).

[6] A. Franciosi, P. Soukassian, P. Philip, S. Chang, A. Wall, A. Raisanen, N. Troullier, Phys. Rev. B 35, 910 (1987); H.I.Starnberg, P. Soukassian, M.II. Bakshi, Z. Hurych, Phys. Rev. B 37, 1315 (1988).

[7] P. Perfetti, C. Quaresima, C. Coluzza, C. Fortunato, G. Margaritondo, Phys. Rev. Lett. 57, 2065 (1986).

[8] S. Lagomarsino, F. Scarinci, P. Castrucci, C. Giannini, E. Fontes, J.R. Patel, Phys. Rev. B 46, 13631 (1992).

[9] K. Takayanagi, Y. Tanishiro, M. Takahashi, S. Takahıashi, J. Vac. Sci. Technol. A 3, 1502 (1985).

[10] G.X. Qian, D.J. Chadi, Phys. Rev. B 35, 1288 (1987).

[11] T. Kendelewicz, P. Soukassian, R.S. List, J.C. Woicik, P. Pianetta, I. Lindau, W.E. Spicer, Phys. Rev. B 37, 7115 (1988).

[12] Y. Hasegawa, I. Kamiya, T. Hashizume, T. Sakurai, II. Tochihara, M. Kubota, Y. Murata, J. Vac. Sci. Technol. A 8, 238 (1990).

[13] R.J. Hammer, R.M. Tromp, J.E. Demuth, Phys. Rev. B 34, 5343 (1986).

[14] C. Giannini, L. Tapfer, S. Lagonarsino, J.C. Boulliard, A. Taccoen, B. Capelle, M. Ilg, O. Brandt, K. Ploog, Phys. Rev. B 48, 11496 (1993).

[15] T.W. Barbee, W.K. Warburton, Mater. Lett. 3, 12 (1984).

[16] A. Iida, T. Matsushita, T. Islikawa, Jpn. J. Appl. Phys. 24, L675 (1989).

[17] S.I. Zheludeva, S. Lagomarsino, N.N. Novikova, M.V. Kovalchuk, F. Scarinci, Thin Solid Films 103/194, 395 (1990).

[18] M.J. Bedzyk, G.M. Bommarito, J.S. Schildkraut, Phys. Rev. Lett. 62, 1376 (1989). 